\title{
Complete nucleotide sequence and annotation of the temperate corynephage $\phi 16$ genome
}

\author{
Juliya S. Lobanova ${ }^{1}$ (D) Evgueni R. Gak ${ }^{1} \cdot$ Irina G. Andreeva $^{1} \cdot$ Konstantin V. Rybak $^{1}$ • \\ Alexander A. Krylov ${ }^{1} \cdot$ Sergey V. Mashko ${ }^{1}$
}

Received: 31 January 2017/ Accepted: 16 March 2017/Published online: 28 April 2017

(c) The Author(s) 2017. This article is an open access publication

\begin{abstract}
The complete genome of $\phi 16$, a temperate corynephage from Corynebacterium glutamicum ATCC 21792, was sequenced and annotated (GenBank: KY250482). The electron microscopy study of $\phi 16$ virion confirmed that it belongs to the family Siphoviridae. The $\phi$ 16 genome consists of a linear double-stranded DNA molecule of 58,200 bp $(\mathrm{G}+\mathrm{C}=52.2 \%)$ with protruding cohesive 3'-ends of 14 nt. Four major structural proteins were separated by SDS-PAGE and identified by peptide mass fingerprinting technique. Using bioinformatics analysis, 101 putative ORFs and 5 tRNA genes were predicted. Only 27 putative gene products could be assigned to known biological functions. The $\phi 16$ genome was divided into functional modules. Seven putative promoters and eight putative unidirectional intrinsic terminators were predicted. One site of putative «-1» programmed ribosomal
\end{abstract}

Electronic supplementary material The online version of this article (doi:10.1007/s00705-017-3383-4) contains supplementary material, which is available to authorized users.

Juliya S. Lobanova

Yulia_Lobanova@agri.ru

Evgueni R. Gak

Evgueni_Gak@agri.ru

Irina G. Andreeva

Irina_Andreeva@agri.ru

Konstantin V. Rybak

Konstantin_Rybak@agri.ru

Alexander A. Krylov

Alex_Krylov@agri.ru

Sergey V. Mashko

Sergey_Mashko@agri.ru

1 Ajinomoto-Genetika Research Institute, 1st Dorozhny pr. 1-1, Moscow 117545, Russian Federation frameshifting was proposed in the phage tail assembly genome region. C. glutamicum genetic tools could be broadened by exploiting the known integrase gene (gp33) and the newly identified excisionase gene (gp47), participating in site-specific recombination between $\phi 16-a t t P /$ $a t t B$.

Corynebacterium glutamicum is widely used to produce commercially interesting bio-based substances [1]. Phages present a problem for the biotechnology industry and cause financial losses. Many corynephages have been isolated, but only a few of them have been completely sequenced (e.g. [2, 3]). In the present study, the genome of $\phi 16$, a temperate corynephage from $C$. glutamicum (ATCC 21792), kindly provided by Dr. Trautwetter [4], was sequenced and annotated. This information could provide valuable evolutionary insights and be helpful for phageresistant strain construction [5]. Different integrative vectors targeting different $a t t B$-sites have been constructed based on known integrases of phages $\phi$ AAU2 [6], beta [7], $\phi 304 \mathrm{~L}$ [8] and $\phi 16$ [9] from C. glutamicum strains. The newly identified $\phi 16$ excisionase, in addition to the known integrase gene, could be useful for broadening C. glutamicum genetic tools, e.g. for site-specific integration/excision of DNA fragments into bacterial chromosomes, as was demonstrated for other phage-based systems [10].

Phage $\phi 16$ was induced from the natural lysogen, $C$. glutamicum ATCC 21792, propagated and purified by centrifugation in $\mathrm{CsCl}$-gradient as described $[4,11]$.

Transmission electron microscopy study of $\phi 16$ virion confirmed that it belongs to the family Siphoviridae, with a polyhedral head of $73 \mathrm{~nm}$ in width and $336 \mathrm{~nm}$ in length, and with a non-contractile striated tail of $14 \mathrm{~nm}$ in diameter (Fig. 1a), in line with Dr. Trautwetter's group data [4]. 
(a)

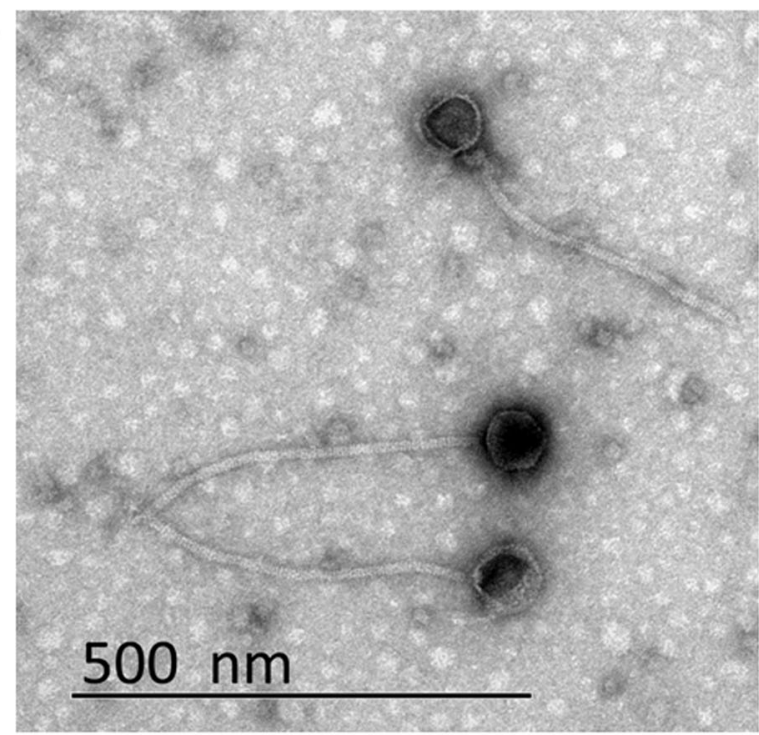

(b)

(c)

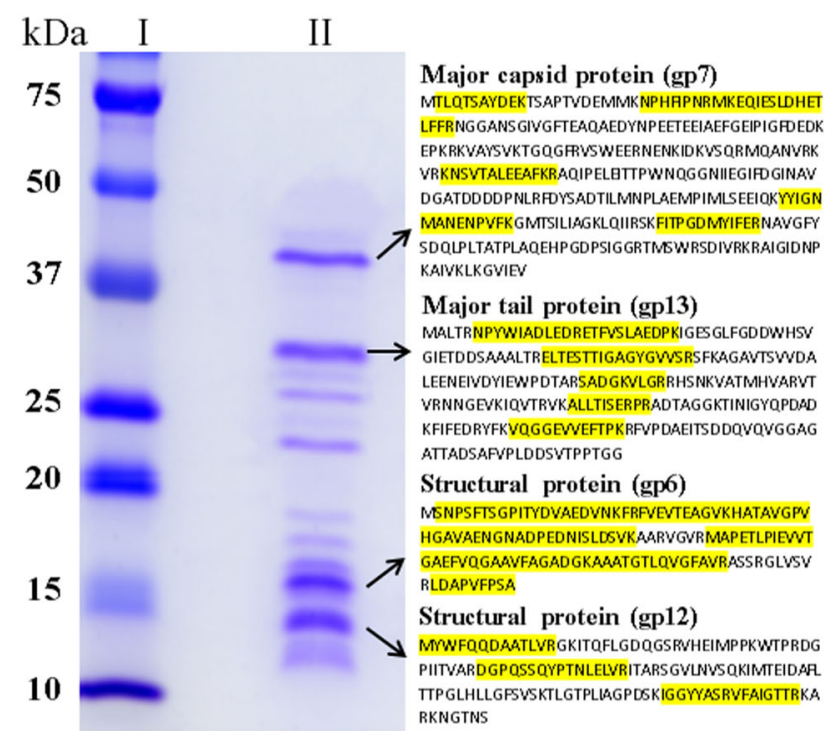

Fig. 1 (a) Electron micrograph of $\phi 16$. Bar, $500 \mathrm{~nm}$. (b) SDS-PAGE analysis of $\phi 16$ structural proteins. Molecular weight markers (lane I). Protein profile of $\phi 16$ (lane II). (c) Four major bands underwent peptide mass fingerprinting analysis; the corresponding predicted amino acid sequences (not highlighted) and the aa sequences detected in the analysis (highlighted) are shown

Subsequently, one of the putative $\phi 16$ gene products (gp), gp16, was assigned to the tail tape measure protein (TMP). The relationship between observed tail length $(\sim 336 \mathrm{~nm})$ and TMP size $(2,151 \mathrm{aa})$, with a ratio of $0.156 \mathrm{~nm} / \mathrm{aa}$, is reasonable [12].

Purified phage DNA was hydrodynamically sheared, and fragments of 2 to $5 \mathrm{~kb}$ in size were blunted and then cloned and sequenced by the Sanger's method. A total of 346 individual DNA fragments were sequenced with an average length of $750 \pm 130 \mathrm{bp}$, and an achieved sequence coverage of $\sim 6$ 6-fold. Closure of gaps was accomplished by primer walking. The genome sequence was finalized by determining the cos sequence with a sequence run-off experiment and comparison of the nucleotide sequence with the ligated phage ends.

Three bioinformatic on-line programs were used for ORFs prediction in the $\phi 16$ genome: Open Reading Frame Finder (https://www.ncbi.nlm.nih.gov/orffinder/), Glimmer 3 (http://www.ncbi.nlm.nih.gov/genomes/MICROBES/gli mmer_3.cgi) and GeneMark S (http://exon.biology.gatech. edu/). The putative tRNAs genes were predicted using tRNAscan-SE (http://lowelab.ucsc.edu/tRNAscan-SE/). Two web services, the phiSITE PromoterHunter (http:// www.phisite.org/main/index.php?nav=tools\&nav_sel=hun ter) (with parameters for " -10 " and " -35 " [Supplementary Fig. 1]) and the PePPER tool-box (http://pepper.molgen rug.nl) were used to search for putative promoters. Intrinsic terminators were identified with "ARNold finding terminators" (http://rna.igmors.u-psud.fr/toolbox/arnold/index. php) with additional evaluation of free energy parameters by the online version of the ViennaRNA package (http:// rna.tbi.univie.ac.at/).

The $\phi 16$ genome is a double-stranded DNA molecule of 58,200 bp in length $(\mathrm{G}+\mathrm{C}=52.2 \%)$ with $3^{\prime}$-protruding single-stranded cohesive ends of 14 nt ( $3^{\prime}$-GGAAGGTGGAGGCT and CCTTCCACCTCCGA-3'). Using bioinformatics analysis, 101 putative ORFs covering $\sim 92.4 \%$ of the total DNA length were identified. Only 27 $\mathrm{gp}(\mathrm{s})$ could be assigned to known biological functions (Supplementary Table 1); the other $55 \mathrm{gp}(\mathrm{s})$ displayed homology to hypothetical proteins, and $19 \mathrm{gp}(\mathrm{s})$ had no homologues in the databases (Fig. 2). Seven putative promoters $(1-$ leftward and 6 - rightward) and eight putative intrinsic unidirectional terminators $(1-$ leftward and $7-$ rightward) were predicted in the intergenic spaces of the $\phi$ 16 genome (Fig. 2, Supplementary Table 2, 3). Five tRNA Lys(UUU), $\operatorname{Arg}(\mathrm{UCU}), \quad \operatorname{Asn}(\mathrm{GUU}), \quad \operatorname{Tyr}(\mathrm{GUA})$ and $\operatorname{Trp}(\mathrm{CCA})$ were identified (Supplementary Fig. 2). Comparison between $\phi 16$ and $C$. glutamicum codons frequency support the hypothesis that phage-encoded tRNAs could compensate codon frequency bias and promote efficient translation of phage-derived mRNA [13] (Supplementary Fig. 3).

Based on homology to known phage proteins, functional domains, and mutual arrangement, putative functions were assigned to products of 27 predicted ORFs (Supplementary Table 1). The entire genome was divided into the six functional modules (Fig. 2). The DNA packaging module contains small and large terminase subunits (gp2 and gp3) and the portal protein (gp4). The prohead protease (gp5), the major capsid and tail proteins (gp7 and gp13), the tail TMP (gp16), and the tail fiber protein (gp20) could be predicted in the structural components and assembly 

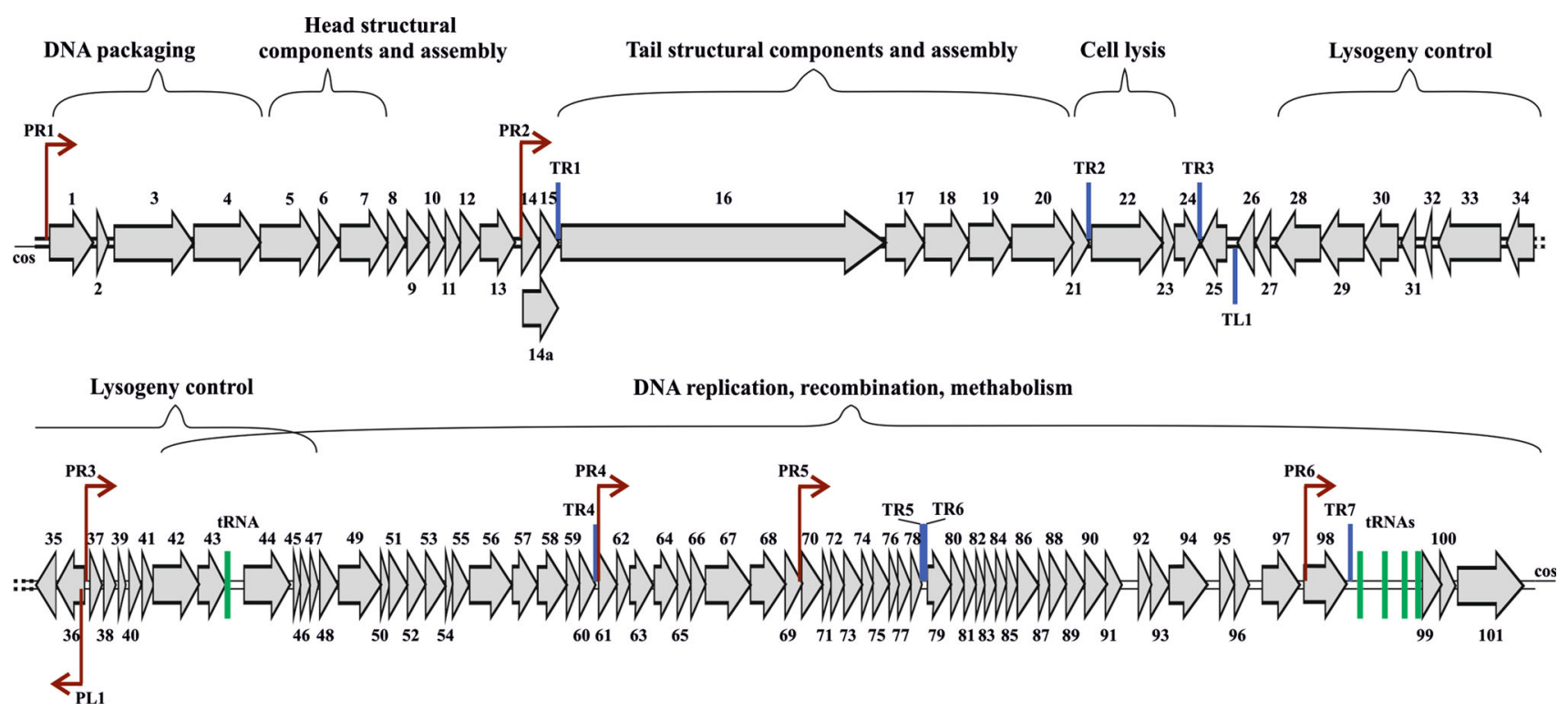

Fig. 2 Genomic organization of $\phi 16$ phage. ORFs are numbered consecutively from left to right and are indicated by arrows (or triangles) in the direction of transcription. ORFs, joined by braces, are provided for the proposed functional modules of the $\phi 16$ genome.

module. At the same time, four major structural proteins, the major capsid and tail proteins (gp7 and gp13) and two proteins with unknown function (gp6 and gp12), were detected by SDS-PAGE and identified by trypsin-based peptide mass fingerprinting technique (PMF), using Ultraflex II LC-MALDI-TOF/TOF (Brucker), performed according to Govorun et al. [14]. Furthermore, detection of $\mathrm{N}$-terminal Met residue retention in a trypsin-digested peptide from gp12 and its elimination from N-terminal peptides from gp6 and gp7 confirmed the $\mathrm{N}$-terminal processing rule [15] (Fig. 1b, c).

A putative site of a -1 programmed ribosomal frameshifting (PRF) could be found in the proposed tail assembly genes and was composed of three functional elements: an internal SD (5'-GAGG $\rightarrow 3$ '), a "slippery sequence" ( $5^{\prime}$-GGGGGAA $\left.\rightarrow 3^{\prime}\right)$ and an H-type pseudoknot RNA structure (Supplementary Fig. 4) [16]. The PRF was predicted to lead to the formation of a large fusion protein, gp14A.

Homologues of two known enzymes were predicted in the host lysis module: the endolysin (gp22) and the holin (gp23). The lysogeny control module was unusual: it contained two putative integrases (gp33 and gp28), the excisionase (gp47), the phage superinfection exclusion protein (gp34), and the transcriptional regulator (gp36). The nucleotide sequence of the $\phi 16$ int gene (corresponding to ORF33 in our annotation) and the $\phi 16$ attP site were deposited previously (GenBank: Y12471.1) [8] and differ from the newly sequenced ORF33 in several points due to sequencing errors in the past, that resulted in
Promoter positions and directions are indicated by thin arrows; intrinsic terminators and tRNAs are depicted as dark and light boxes, respectively

differences in the structures of the corresponding gp(s) (Supplementary Fig. 5). We confirmed the ability of gp33 to provide site-specific integration of recombinant DNA into the $\phi 16-a t t B$ of the $C$. glutamicum ATCC 21792c chromosome, which was previously shown by the Trautwetter group [9]. We also demonstrated experimentally the effective excision of integrated recombinant DNA when gp33 and gp47 are expressed simultaneously of (manuscript in preparation). The experiments also showed that the second putative integrase, gp28, could not use the previously established $\phi 16$-attP site [8] for site-specific recombination. No other putative $a t t P$-site was detected in the vicinity of ORF28 (unpublished result).

The replication/recombination/metabolism module also contained homologues to known proteins: ParB-like protein (gp42), HNH homing endonuclease (gp52), the transcriptional regulator (gp56), SSB protein (gp58), glutaredoxin (gp62), RusA endodeoxyribonuclease (gp63), methyltransferase (gp64), chromosomal partitioning protein (gp68), oligoribonuclease (gp94) and ATPase (gp101).

The analysis indicated that some modules of the $\phi 16$ genome had complete or partial homology to distinct chromosomal regions of four bacteria, leading us to hyphothesize that these are uncharacterized prophages in bacterial genomes. Throughout large parts of the genome sequence, significant similarity was observed between $\phi 16$ and the hypothetical prophage Corynebacterium pyruviciproducens ATCC BAA-1742, at the nucleotide and deduced protein sequence level. Significant similarity was also observed, throughout the whole genome, between $\phi 16$ 
and the hypothetical prophage Brevibacterium flavum ATCC 15168 (identical to prophage of C. glutamicum ATCC 14067). We also observed similarity between predicted $\phi 16$ proteins, which are involved in DNA packaging, head and tail structural components, as well as assembly modules, and proteins of a Corynebacterium ulcerans BR-AD22 hypothetical prophage. Several $\phi 16$ lysogeny control genes (ORFs $30,33,35,47$ ) were very similar, at the protein and nucleotide level, to genes of the hypothetical prophage of $C$. falsenii DSM 44353, strain BL 8171. Furthermore, a significant part of the $\phi 16$ genome, as well as the second part of the lysogeny control genes (ORFs 28, 29, 34, 36) share homology with a hypothetical prophage from $C$. pyruviciproducens ATCC BAA-1742. To demonstrate the general sequence homology with the four hypothetical prophages, multi-dot plots were obtained (Supplementary Fig. 6). Neither protein nor nucleotide homology was observed between $\phi 16$ and BFK20 or P1201. However, the $\phi 16$ genome is not present in any bacterial chromosome in the database; therefore, the sequence of the entire $\phi 16$ phage genome was deposited for the first time in GenBank, under accession number KY250482.

Acknowledgements The authors are very grateful to Drs. Andrey O. Lobanov, Sergey V. Smirnov, and Yurges A.V. Yomantas for helpful discussions.

\section{Compliance with ethical standards}

Conflict of interest There is no conflict of interest.

Ethical approval This article does not contain any studies with human participants or animals performed by any of the authors.

Open Access This article is distributed under the terms of the Creative Commons Attribution 4.0 International License (http://crea tivecommons.org/licenses/by/4.0/), which permits unrestricted use, distribution, and reproduction in any medium, provided you give appropriate credit to the original author(s) and the source, provide a link to the Creative Commons license, and indicate if changes were made.

\section{References}

1. Becker J, Wittmann C (2012) Bio-based production of chemicals, materials and fuels-Corynebacterium glutamicum as versatile cell factory. Curr Opin Biotechnol 23:631-640. doi:10.1016/j. copbio.2011.11.012

2. Bukovska G, Klucar L, Vlcek C, Adamovic J, Turna J, Timko J (2006) Complete nucleotide sequence and genome analysis of bacteriophage BFK20 - a lytic phage of the industrial producer Brevibacterium flavum. Virology 348:57-71. doi:10.1016/j.virol. 2005.12.010

3. Chen CL, Pan TY, Kan SC et al (2008) Genome sequence of the lytic bacteriophage P1201 from Corynebacterium glutamicum NCHU 87078: Evolutionary relationships to phages from $\mathrm{Co}$ rynebacterineae. Virology 378:226-232. doi:10.1016/j.virol. 2008.05.027

4. Moreau S, Leret V, Marrec CL, Varangot H, Ayache M, Bonnassie S, Blanco C, Trautwetter A (1995) Prophage distribution in coryneform bacteria. Res Microbiol 146:493-505. doi:10.1016/ 0923-2508(96)80295-6

5. Labrie SJ, Samson JE, Moineau S (2010) Bacteriophage resistance mechanisms. Nat Rev Microbiol 8(5):317-327. doi:10. 1038/nrmicro2315

6. LeMarrec C, Moreau S, Loury S, Blanco C, Trautwetter A (1996) Genetic characterization of site-specific integration functions of $\phi$ AAU2 infecting "Arthrobacter aureus" C70. J Bacteriol 178:1996-2004

7. Oram M, Woolston JE, Jacobson AD, Holmes RK, Oram D (2007) Bacteriophage-based vectors for site-specific insertion of DNA in the chromosome of Corynebacteria. Gene 391(1-2):53-62. doi:10.1016/j.gene.2006.12.003

8. Moreau S, Le Marrec C, Blanco C, Trautwetter A (1999) Analysis of the integration functions of \& \#x03D5;304L: an integrase module among corynephages. Virology 255:150-159. doi:10. 1006/viro.1998.9524

9. Moreau S, Blanco C, Trautwetter A (1999) Site-specific integration of corynephage $\phi 16$ : construction of an integration vector. Microbiology 145:539-548. doi:10.1099/13500872-145-3539

10. Haldimann A, Wanner BL (2001) Conditional-replication, integration, excision, and retrieval plasmid-host systems for gene structure-function studies of bacteria. J Bacteriol 183:6384-6393. doi:10.1128/JB.183.21.6384-6393.2001

11. Boulanger P (2009) Purification of bacteriphages and SDS-PAGE analysis of phage structural proteins from host particles. Methods Mol Biol 502:227-238. doi:10.1007/978-1-60327-565-1_13

12. Abuladze NK, Gingery M, Tsai J, Eiserling FA (1994) Tail length determination in bacteriophage T4. Virology 199(2):301-310. doi:10.1006/viro.1994.1128

13. Bailly-Bechet M, Vergassola M, Rocha E (2007) Causes for the intriguing presence of tRNAs in phages. Genome Res 17:1486-1495. doi:10.1101/gr.6649807

14. Govorun VM, Moshkovskii SA, Tikhonova OV et al (2003) Comparative analysis of proteome maps of Helicobacter pylori clinical isolates. Biochemistry (Mosc) 68:42-49

15. Eggeling L, Bott M (2005) Handbook of Corynebacterium glutamicum. CRC Press, Boca Raton

16. Xu J, Hendrix RW, Duda RL (2004) Conserved translational frameshift in dsDNA bacteriophage tail assembly genes. Mol Cell 16(1):11-21. doi:10.1016/j.molcel.2004.09.006 